Ciclos biogeoquímicos y fertilidad del suelo

\title{
Impacto de la siembra directa en la estabilización de la materia orgánica frente al avance de la frontera agrícola
}

${ }^{1}$ Koritko Lucas, ${ }^{2}$ Suárez Ariel, ${ }^{2}$ Albanesi Ada.

${ }^{1}$ Becario CONICET

${ }^{2}$ FAyA, UNSE. Av. Belgrano (S) 1912. Santiago del Estero. CP 4200

*E-mail: lucaskoritko@gmail.com, albanesi@unse.edu.ar

Introducción: El cambio del uso del suelo y la necesidad de mejorar la sostenibilidad de las prácticas agrícolas ha llevado a la adopción generalizada de los procesos de mínimo impacto como la agricultura bajo siembra directa (ABSD) que ayudan a preservar y/o acumular la materia orgánica del suelo (MOS). Los objetivos de este trabajo fueron describir y explicar a escala de sitio los cambios de estado que suceden en el ecosistema monte por la intervención agrícola con siembra directa y conocer la dinámica de fracciones de carbono y nitrógeno del suelo en sistemas agrícolas con siembra directa en sitios de la ecoregión chaco semiárido.

Materiales y Métodos: El área de estudio estuvo comprendida en el Centro-Este de la provincia de Santiago del Estero, Argentina (área con mayor tasa de desmontes). En un Diseño en bloques completos al azar se localizaron 3 campos (bloques) con suelos de lotes que provenían de diferentes años de siembra directa $(0,3,6,10$ y 17) a partir del desmonte; se midió carbono orgánico total (COT), carbono orgánico particulado (COP), nitrógeno total (NOT) y particulado (NOP) tomando muestras a dos profundidades de $0-5 \mathrm{~cm}(\mathrm{P} 1)$ y $5-20 \mathrm{~cm}(\mathrm{P} 2)$. Cada variable se analizó con un enfoque de modelos mixtos con el programa $\mathrm{R}$ a través de Infostat.

Resultados: Las profundidades de muestreo y el manejo de suelo fueron considerados efectos fijos mientras que los bloques se asociaron a efectos aleatorios. La comparación de medias se efectuó usando la prueba de DGC con un nivel de confianza del $95 \%$. Los valores de COT, COP, NOT y NOP redujeron marcadamente una vez producido el disturbio. En función de la profundidad se hallaron diferencias significativas siendo la P1 quien mejor administra el recurso en todas las variables. El COT disminuyó 52 $\%$, mientras que el COP bajó $67 \%$ luego de 17 años de ABSD respecto del monte en los primeros $5 \mathrm{~cm}$ de profundidad. Sin embargo, esto significó solo un agotamiento del $15 \%$ (COT) y $20 \%$ (COP) en relación a los 3 años bajo este manejo. Con pérdidas del $53 \%$ (NOT) y $48 \%$ (NOP) existió similar comportamiento, las cuales representan entre el $20 \%$ y $16 \%$ respectivamente.

Tabla.Valores promedio de carbono orgánico total (COT), carbono particulado (COP), nitrógeno total (NOT), nitrógeno particulado (NOP) y su respuesta al cambio de uso del suelo y profundidad.

\begin{tabular}{|c|c|c|c|c|c|}
\hline & \multicolumn{3}{|c|}{ Fracciones de Carbono } & \multicolumn{2}{|c|}{ Fracciones de Nitrógeno } \\
\hline ABSD & Profundidad & COT & COP & NOT & NOP \\
\hline \multirow{2}{*}{ SD - 0} & $\mathrm{P} 1$ & 38,39 a & $19,75 \mathrm{a}$ & $4,19 \mathrm{a}$ & $2,42 \mathrm{a}$ \\
\hline & $\mathrm{P} 2$ & $24,79 \mathrm{~b}$ & $6,23 \mathrm{~b}$ & $2,05 \mathrm{~b}$ & $1,15 \mathrm{~b}$ \\
\hline \multirow{2}{*}{ SD - 3} & $\mathrm{P} 1$ & $21,86 \mathrm{~b}$ & $8,49 \mathrm{~b}$ & $2,46 \mathrm{~b}$ & $1,51 \mathrm{~b}$ \\
\hline & $\mathrm{P} 2$ & $17,76 \mathrm{~b}$ & $2,23 \mathrm{c}$ & $0,74 \mathrm{c}$ & $0,37 \mathrm{c}$ \\
\hline \multirow{2}{*}{ SD - 6} & $\mathrm{P} 1$ & $18,44 \mathrm{~b}$ & $7,06 \mathrm{~b}$ & $2,36 \mathrm{~b}$ & $1,71 \mathrm{~b}$ \\
\hline & $\mathrm{P} 2$ & $12,09 \mathrm{c}$ & $2,92 \mathrm{c}$ & $0,99 \mathrm{c}$ & $0,64 \mathrm{c}$ \\
\hline \multirow{2}{*}{ SD - 10} & $\mathrm{P} 1$ & $15,16 \mathrm{~b}$ & $7,28 \mathrm{~b}$ & $2,14 \mathrm{~b}$ & $1,13 \mathrm{~b}$ \\
\hline & $\mathrm{P} 2$ & $11,06 \mathrm{c}$ & $1,45 \mathrm{c}$ & $1,10 \mathrm{c}$ & $0,55 \mathrm{c}$ \\
\hline \multirow{2}{*}{ SD - 17} & $\mathrm{P} 1$ & $18,56 \mathrm{~b}$ & $6,78 \mathrm{~b}$ & $1,97 \mathrm{~b}$ & $1,27 \mathrm{~b}$ \\
\hline & $\mathrm{P} 2$ & $10,98 \mathrm{c}$ & $3,14 \mathrm{c}$ & $1,07 \mathrm{c}$ & $0,58 \mathrm{c}$ \\
\hline
\end{tabular}

Referencias $: A B S D=$ años agricultura bajo siembra directa $: P 1=$ profundidad $=0-5 \mathrm{~cm} ; P 2=$ profundidad $5-20 \mathrm{~cm}$. Medias con letra distinta indican diferencias estadísticamente significativas $(p<0,05)$.

Conclusión: En este trabajo se demostró que el reemplazo del monte sugiere un importante cambio en la degradación del $\mathrm{C}$ y $\mathrm{N}$ al pasar al manejo agrícola. Sin embargo, es de destacar que no se modifican de manera significativa los valores una vez implementada esta práctica de mínimo impacto, haciendo de ésta un manejo sustentable en el tiempo preservando y estabilizando la MOS.

Palabras clave: Siembra Directa, C y N total, C y N particulado

Agradecimientos: Al Ing. Agr. Ariel Herrero y el señor Nicolás Darling 